Colman, A. M., Best, W. M., \& Austen, A. J. (1986). Familiarity and liking: Direct tests of the preferencefeedback hypothesis. Psychological Reports, 58, 931-938.

\title{
Familiarity and Liking: Direct Tests of the Preference-Feedback Hypothesis ${ }^{1}$
}

\author{
Andrew M. Colman, ${ }^{2}$ Wendy M. Best, and Alison J. Austen
}

\author{
University of Leicester
}

\begin{abstract}
Summary.-
Previous investigations have provided evidence for positive (“mere exposure”), negative, and inverted-U functional relationships between familiarity and liking for various categories of stimuli. The preferencefeedback hypothesis offers an explanation for these seemingly contradictory findings; two experiments designed to test the hypothesis directly are reported in this paper. In both experiments, as predicted by the hypothesis, mere exposure effects were found for Class A stimuli, whose cultural prevalence is determined partly by their popularity; but the hypothesized nonmonotonic familiarity-liking relationship did not emerge for Class $B$ stimuli, whose cultural prevalence is unresponsive to their popularity. Four possible explanations of these findings are discussed.
\end{abstract}

In a classic monograph, Zajonc (1968) reviewed correlational, quasiexperimental, and experimental evidence bearing on the hypothesis that "mere repeated exposure of the individual to a stimulus is a sufficient condition for the enhancement of his attitude toward it" (p. 1). Since the publication of Zajonc's monograph, a considerable volume of additional evidence (Harrison, 1977; Sluckin, Hargreaves, \& Colman, 1983; Stang, 1974) has accumulated in support of the mere exposure hypothesis, as it is called, but some contradictory evidence has also come to light. Cantor (1968) and Cantor and Kubose (1969) reported that children gave more favorable ratings to unfamiliar than to familiar geometric figures taken from the Welsh Figure Preference Test. Berlyne (1970) found that certain simple works of art were rated as progressively less pleasing by adult subjects as exposure frequency increased. Faw and Pien (1971) found that both children and adults preferred novel to familiar line drawings of common objects and simple nonrepresentational patterns. Siebold's (1972) young subjects also preferred unfamiliar geometric patterns to the same patterns after repeated experimental exposure.

To complicate the picture further, a number of investigations reviewed by Sluckin, Hargreaves, and Colman (1983) have reported an initial increase in liking after familiarization followed by a subsequent decrease in liking after further familiarization for various classes of stimuli. These findings led Berlyne (1971) to propose an inverted-U hypothesis (based on an old idea of Wundt's) concerning the functional relationship between familiarity and liking. The great advantage of the inverted-U hypothesis--that it can account for positive (Zajonc-type), negative (Cantor-type), and nonmonotonic familiarity-liking correlations--is also its greatest drawback: it is difficult to specify any set of observations that would clearly refute it. Since the inverted-U hypothesis may nonetheless be true as far as it goes, it seemed to us that what was needed was a theoretical development that would render it testable, at least in part. Therefore, on the basis of two empirical investigations which, in spite of almost identical design and methodology, gave seemingly contradictory results, 
Colman, Sluckin, and Hargreaves (1981) proposed a preference-feedback hypothesis, which was intended to be directly testable.

The first investigation (Colman, Hargreaves, \& Sluckin, 1981) consisted of two experiments, one conducted in England and one in Australia, on familiarity and liking for 100 randomly selected Christian names. In both experiments, strong positive correlations were found between familiarity and liking for both male and female names, whether judged by men or women, and there was no evidence of any curvilinear trend in the data. The second investigation (Colman, Sluckin, \& Hargreaves, 1981) was an almost exact replication of the first, except that the stimuli were 60 randomly selected surnames. In this case, however, the best-liked names turned out to be those of intermediate familiarity, and a regression analysis confirmed the fact that a large and significant proportion of the variance in ratings was attributable to an incremental quadratic (inverted-U) component.

The fundamental assumption of the preference-feedback hypothesis is that naturally occurring stimuli can be divided into two classes, or (as we should prefer to put it now) that they fall on a continuum between two ideal types. Class $A$ contains elements whose cultural prevalence is responsive to their popularity--when they are generally liked people tend to be exposed to them relatively frequently, and if they start to become disliked (perhaps as a result of overexposure, which results in satiation and boredom), their frequency of occurrence in the culture decreases, thereby forestalling any further decline in popularity through exposure.

Clear examples of Class $A$ stimuli include popular tunes and fashion clothes, and Christian names also belong to Class $A$ because those that are generally disliked are given to newborn infants less frequently than popular names. According to the preference-feedback hypothesis, Class $A$ stimuli are prevented by a cybernetic feedback mechanism from progressing along the descending part of the inverted-U. Therefore, for these stimuli, positive, monotonic familiarity-liking relationships are predicted. Class $B$, on the other hand, contains stimuli whose cultural prevalence is more or less unresponsive to their popularity: letters of the alphabet and digits are good examples, and surnames clearly belong to this class also because they are seldom chosen voluntarily--their frequency of occurrence in the culture is almost wholly determined by factors other than esthetic preference. There is no preference-feedback mechanism to prevent the most familiar of the Class $B$ stimuli from continuing to be overexposed and therefore to progress along the descending part of the inverted-U. Nonmonotonic familiarity-liking relationships are predicted in these cases.

The preference-feedback hypothesis appears to account for the seemingly contradictory results of previous experiments on Christian names and surnames (Colman, Hargreaves, \& Sluckin, 1981; Colman, Sluckin, \& Hargreaves, 1981) and for most of the published findings on familiarity and liking for other classes of naturally occurring stimuli. The purpose of the two experiments described below is to test the hypothesis directly, by comparing responses to selected representatives of Class $A$ and Class $B$ stimuli.

\section{Experiment 1}

\section{Method}

The subjects were 22 male and 22 female undergraduate students at the University of Leicester, randomly assigned to rate 48 line drawings mounted on 6 -in. $x$ 4-in. cards either 
for familiarity ("how often you have seen something like it") or for liking ("esthetic appreciation of the object represented in the drawing and not the appeal of the drawing itself") on five-point scales from "unfamiliar" or "dislike" to "familiar" or "like". Half the objects depicted in the drawings were selected on commonsense grounds as probable representatives of Class A: hats, shoes, dresses, and hairstyles. Each category contained six stimuli, ranging from the commonplace to the distinctly unusual. The other half represented putative Class $B$ stimuli in the following categories: houses, clouds, land masses, and trees, with once again six examples, ranging from common to uncommon, in each category. Subjects were tested individually, and the deck of 48 cards was thoroughly shuffled before each presentation.

\section{Results}

For the Class $A$ stimuli, linear regression analysis yielded the least-squares line defined by $\mathrm{FAV}(A)=1.11+0.27 \mathrm{FAM}(A)$, where $\mathrm{FAV}(A)$ and $\mathrm{FAM}(A)$ are the mean ratings of liking (favorability) and familiarity respectively for the Class $A$ stimuli. The coefficient of determination is $R^{2} \mathrm{FAV}(A) \cdot \operatorname{FAM}(A)=0.256$.

For Class $B, \operatorname{FAV}(B)=1.24+0.45 \mathrm{FAM}(B)$, where $\operatorname{FAV}(B)$ and $\operatorname{FAM}(B)$ are the mean liking and familiarity ratings for Class $B$. The coefficient of determination is $R^{2} \operatorname{FAV}(B)$. $F A M(B)=$ 0.504 .

The significance of the linear component was tested in each case by evaluating

$$
F(k, n-k-1)=\left[R^{2} \mathrm{FAV} . \mathrm{FAM} / k\right] /\left[\left(1-R^{2} \mathrm{FAV} . \mathrm{FAM}\right) /(n-k-1)\right],
$$

where $n$ is the number of stimuli and $k$ is the degrees of freedom for $R^{2}$ FAV.FAM. For Class $A, n=24, k=1$, and $F(1,22)=7.57, \mathrm{p}<0.02$.

For Class $B, n=24, k=1$, and $F(1,22)=22.40, p<0.001$. Quadratic regression analysis yielded the following least-squares parabolas:

$$
\operatorname{FAV}(A)=0.42+1.23 \mathrm{FAM}(A)-0.24 \mathrm{FAM}(A)^{2}
$$

with coefficient of determination $R^{2} \operatorname{FAV}(A) \cdot \operatorname{FAM}(A), \operatorname{FAM}(A)^{2}=0.381$, and

$$
\operatorname{FAV}(B)=.055+1.19 \mathrm{FAM}(B)-0.17 \mathrm{FAM}(B)^{2} \text {, }
$$

with coefficient of determination $R^{2} \operatorname{FAV}(B) \cdot \operatorname{FAM}(B), \operatorname{FAM}(B)^{2}=0.553$. The significance of the incremental variance accounted for by the quadratic component over and above the linear component was found by evaluating

$$
F\left(k_{1}-k_{2}, n-k-1\right)=\left(R^{2} \mathrm{FAV} \cdot \mathrm{FAM}, \mathrm{FAM}^{2}-R^{2} \mathrm{FAV} \cdot \mathrm{FAM}\right) /\left(k_{1}-k_{2}\right)
$$

where $k_{1}$ and $k_{2}$ are degrees of freedom for $R^{2}$ FAV.FAM, FAM ${ }^{2}$ and $R^{2}$ FAV.FAM, respectively. For Class $A$ stimuli, $k_{1}=2, k_{2}=1$, and $F(1,21)=4.24$ (n.s.). For Class $B, k_{1}=2$, $k_{2}=1$, and $F(1,21)=2.30$ (n.s.).

These results cannot be said to confirm the preference-feedback hypothesis. Class $A$ stimuli 
manifested the monotonic familiarity-liking relationship that is predicted by both the preference-feedback and mere exposure hypotheses. The linear trend was also significant for Class $B$ stimuli, and no significant incremental quadratic trend was found among Class $B$ stimuli as predicted by the preference-feedback hypothesis alone.

A visual inspection of the data suggested widely differing familiarity-liking relationships for the four categories of Class $B$ stimuli (houses as compared with trees, for example) but with only six examples in each category it was impracticable to perform separate regression analyses within categories. To throw further light on these puzzling results, a second experiment was designed with more examples in each category.

\section{Experiment 2}

This second experiment was similar to the first in design and methodology, but completely new sets of Class $A$ and Class $B$ stimuli were devised, and a larger number of examples was presented in each stimulus category.

\section{Method}

The subjects were 41 men and 19 women, mostly undergraduate students at the University of Leicester, assigned randomly to treatment conditions and tested as in the first experiment. The stimuli were presented in the form of brief verbal descriptions rather than pictorially. There were three categories of Class $A$ stimuli, with 20 examples in each category: leisure activities ("listening to records", "writing poetry”, etc.); foods ("roast beef”, "moussaka”, etc.); and dogs' names ("Rex”, "Shep”, etc.).

There were also three categories of Class $B$ stimuli with 20 examples in each category: names of towns and cities ("Edinburgh”, "Grantham”, etc.); combinations of hair and eye color in men ("red hair, brown eyes", "black hair, green eyes", etc.); and speech sounds ("g as in green", "i as in pin", etc). The reason for restricting the second Class B category to hair and eye color in men only was that men do not normally alter the colors they are endowed with by nature, whereas among women hair color is partly determined by voluntary choice. Each subject rated all the stimuli for either familiarity or liking on five-point scales with the same anchors as in the first experiment.

\section{Results}

The results were analysed in the manner described above. Taking all Class $A$ stimuli together, the linear component of the familiarity-liking relationship was fairly strong $\left(R^{2}=0.251\right)$ and significant, $F(1,58)=19.44, \mathrm{p}<0.001$. The incremental variance accounted for by the quadratic component was negligible, $F(1,57)=1.17$, n.s.). These findings, which replicate those of the previous experiment, are consistent with both the preference-feedback and mere exposure hypotheses.

Supplementary analyses performed on each category of Class $A$ stimuli separately indicated, however, that the linear component accounted for a substantial proportion of the variance $\left(R^{2}\right.$ $=0.411$ ) and was significant, $F(1,18)=12.56 . \mathrm{p}<0.01)$ only for the category of leisure activities. 
The categories of dogs' names and foods yielded weak $\left(R^{2}=0.195\right.$ and $R^{2}=0.106$, respectively) and nonsignificant-- $F(1,18)=4.36$ and $F(1,18)=2.13$, respectively--linear components. The incremental quadratic components were negligible in all categories, as predicted.

For all Class $B$ stimuli taken together, the linear component was very weak $\left(R^{2}=0.041\right)$ and nonsignificant-- $F(1,57)=2.43-$-which is consistent with the hypothesis, but the incremental quadratic component was also very weak and nonsignificant-- $F(1,56)=0.06$--which is not in line with predictions although it once again replicates the results of the first experiment.

Supplementary analyses of individual categories of Class $B$ stimuli gave one case, the category of combinations of hair and eye color in men, in which the linear component was strong $\left(R^{2}=0.494\right)$ and significant-- $F(1,18)=17.57, \mathrm{p}<0.01$. In the other two categories of Class $B$ stimuli the linear component was very weak and nonsignificant, and contrary to the prediction of the preference-feedback hypothesis, the incremental quadratic component was nonsignificant in all three categories: speech sounds, combinations of hair and eye color in men, and names of towns and cities yielded $F$ ratios of $2.78,0.41$, and 0.14 , respectively.

\section{Discussion}

The results of the two experiments reported here do not corroborate the preference-feedback hypothesis. As predicted by the hypothesis, Class A stimuli tended to display the usual monotonic mere exposure effect, although there were two categories of Class $A$ stimuli in the second experiment in which the effect was not significant. The predicted nonmonotonic familiarity-liking relationship among Class $B$ stimuli did not emerge in either experiment, and in the second experiment was not manifest in any of the three categories of Class $B$ stimuli.

There are several possible explanations for the non-emergence of the predicted inverted-U among Class $B$ stimuli. First, the preference-feedback hypothesis may be false, which would imply that one or more of its assumptions is false. The most likely candidate is the assumption that when there is no feedback control mechanism limiting exposure levels, it is the stimuli of intermediate familiarity that are best liked. Given the wealth of evidence (Berlyne, 1970, 1971; Stang, 1974, 1975) concerning the effects of satiation and boredom on esthetic preferences, we are reluctant to abandon this assumption without compelling evidence.

Secondly, it is possible that the preference-feedback hypothesis is sound, but that it was not adequately tested (for Class $B$ stimuli) in the experiments reported here. If this is the case, then the data presented above certainly do not refute the hypothesis. One possibility is that an insufficiently wide range of familiarity was represented among the various categories of Class $B$ stimuli used; this would effectively have truncated the underlying inverted-U and resulted in the nonsignificant quadratic trends observed. Another possibility is that the preference-feedback hypothesis is applicable only to certain classes of stimuli (names, for example) whose popularity is strongly determined by familiarity; the stimuli reported in the experiments reported here may be ones in which preferences are largely determined by factors unrelated to familiarity. This would explain the very high error variance found in most categories of stimuli. Finally, it is possible that the putative Class $B$ stimuli used in our experiments were not true representatives of Class $B$. It is conceivable, taking the categories 
used in our second experiment for illustrative purposes, that there is a preference-feedback mechanism preventing cultural overexposure even of names of towns and cities, combinations of hair and eye color in men, and speech sounds. This last possibility seems rather far-fetched, however, and it would be difficult to explain why the familiarity-liking relationship for names of towns and cities should not follow the inverted-U pattern found previously (Colman, Sluckin, \& Hargreaves, 1981) among surnames.

The results of the experiments reported here provide further evidence in support of Zajonc's (1968) mere exposure hypothesis. But that hypothesis does not explain the negative and nonmonotonic familiarity-liking relationships among certain categories of stimuli that have been reported by several researchers. In particular, it does not explain why the familiarityliking relationship is monotonic for Christian names (Colman, Hargreaves, \& Sluckin, 1981) and nonmonotonic for surnames (Colman, Sluckin, \& Hargreaves, 1981). The preferencefeedback hypothesis offers a possible explanation for all of these effects, including the mere exposure effect. The experiments reported here did not corroborate the preference-feedback hypothesis, but, for the reasons explained above, it would seem premature to conclude that the hypothesis is false, at least until an alternative that can explain all of the reported effects is put forward.

\section{References}

Berlyne, D. E. (1970) Novelty, complexity, and hedonic value. Perception and Psychophysics, 8, 279-286.

Berlyne, D. E. (1971) Aesthetics and psychobiology. New York: Appleton-Century-Crofts.

Cantor, G. N. (1968) Children’s “like-dislike” ratings of familiarized and nonfamiliarized visual stimuli. Journal of Experimental Child Psychology, 6, 651-657.

Cantor, G. N., \& Kubose, S. K. (1969) Preschool children’s ratings of familiarized and nonfamiliarized visual stimuli. Journal of Experimental Child Psychology, 8, 74-81.

Colman, A. M., Hargreaves, D. J., \& Sluckin, W. (1981) Preferences for Christian names as a function of their experienced familiarity. British Journal of Social Psychology, 20, 3-5.

Colman, A. M., Sluckin, W., \& Hargreaves, D. J. (1981) The effect of familiarity on preferences for surnames. British Journal of Psychology, 72, 363-369.

Faw, T. T., \& Pien, D. (1971) The influence of stimulus exposure on rated preference: effects of age, pattern of exposure, and stimulus meaningfulness. Journal of Experimental Child Psychology, 11, 339-346.

Harrison, A. A. (1977) Mere exposure. Advances in Experimental Social Psychology, 10, 3983.

Siebold, J. R. (1972) Children's rating responses as related to amount and recency of stimulus familiarization and stimulus complexity. Journal of Experimental Child Psychology, 14, 257-264. 
Sluckin, W., Hargreaves, D. J., \& Colman, A. M. (1983) Novelty and human aesthetic preferences. In J. Archer \& L. Birke (Eds.), Exploration in animals and humans.

Wokingham: Van Nostrand Reinhold. Pp. 245-269.

Stang, D. J. (1974) Methodological factors in mere exposure research. Psychological Bulletin, 81, 1014-1025.

Stang, D. J. (1975) Effects of “mere exposure” on learning and affect. Journal of Personality and Social Psychology, 31, 7-12.

Zajonc, R. B. (1968) Attitudinal effects of mere exposure. Journal of Personality and Social Psychology, Monograph Supplement, 9(2), Part 2.

\section{Footnotes}

1 The research reported in this paper was supported by Grant C0023 0085 from the Economic and Social Research Council.

2 Requests for reprints should be addressed to Andrew M. Colman, Aesthetics Research Group, Department of Psychology, University of Leicester, Leicester LE1 7RH, England. Wendy Best and Alison Austen are graduates of the Department of Psychology, University of Leicester. 